\title{
Flu transcription captured in action
}

\author{
Viral mRNA synthesis is an essential step in the influenza virus replication cycle and is a prime target for the \\ development of new antivirals. New structures of the influenza virus RNA polymerase now unveil previously \\ unknown details of influenza virus transcription.
}

\author{
Aartjan J. W. te Velthuis
}

n nfluenza virus types A, B and C are human pathogens that cause mild to deadly respiratory infections. For expression of influenza virus proteins during infection, the viral RNA polymerase needs to transcribe the segmented genome of the influenza virus into mRNAs containing a $5^{\prime}$ cap-1 structure and a $3^{\prime}$ poly(A) tail ${ }^{1,2}$. However, unlike those of other RNA viruses ${ }^{3}$, the genomes of influenza viruses do not encode molecules with capping activities. Instead, the influenza virus RNA polymerase starts viral transcription using capped primers derived from host-cell pre-mRNAs ${ }^{4}$. Kouba, Drncova and Cusack now reveal how the influenza B virus RNA polymerase starts the process of turning 'snatched primers' into influenza virus mRNAs ${ }^{5}$.

The influenza virus RNA polymerase is composed of the viral proteins PB1, PB2 and PA. PB1, the $\mathrm{N}$ terminus of PB2 and the C-terminal domain of PA form the core of the polymerase (Fig. 1, stage 1). They are responsible for interacting with the terminal ends of viral promoters (Fig. 1, stage 2) and for catalyzing the nucleotidyltransfer reaction. The core also binds the Ser5-phosphorylated C-terminal domain of initiating cellular polymerase II complexes ${ }^{6,7}$ in order to bring the polymerase into the vicinity of a host pre-mRNA (Fig. 1, stage 3). Capped primers are generated by the flexible PB2 C-terminal domain and PA N-terminal domain, which bind capped pre-mRNAs and cleave capped pre-mRNAs, respectively ${ }^{8}$ (Fig. 1, stage 3).

Typical influenza virus primers are 10-14 nucleotides long and form $1-$ to 3 -base pair interactions with the $3^{\prime}$ terminus of the viral genome segment, depending on the sequence of the primer $^{9}$ (Fig. 1, stage 4). Previously determined crystal structures ${ }^{8,10}$ show how the template and a capped primer are threaded into the core of the RNA polymerase (Fig. 1, stage 4). However, owing to the flexibility of the

$3^{\prime}$ terminus of the viral promoter ${ }^{11,12}$ (Fig. 1, stage 2 ) and limited base pairing between template and capped primer, transcriptioninitiation complexes have proven refractory to crystallization ${ }^{10}$.
Kouba, Drncova and Cusack cleverly overcome that problem by extending the $3^{\prime}$ end of the template by three bases ${ }^{5}$ and thus increasing the number of base pairs that a capped primer and the viral template can form. Using this approach, they capture how the $3^{\prime}$ end of a 14-nucleotide-long primer is coordinated by the RNA polymerase and bound to the -1 base of the template (Fig. 1, stage 5 ). This puts the primer in a position that is ready for extension. Indeed, addition of CTP, which is complementary to the +1 base of the template, to the pre-initiation crystal yields a density opposite the +1 guanosine (Fig. 1, step 6). The structure is, however, in a pre-catalysis state, as only one magnesium ion of the requisite two is present.

To mimic the addition of CTP, Kouba, Drncova and Cusack use a 15-nucleotidelong primer that ends in a cytosine ${ }^{5}$ (Fig. 1, stage 7). This cytosine binds to the +1 guanosine and is accompanied by a pyrophosphate in the location of the triphosphate of the pre-catalysis CTP. Since the template has not translocated relative to stage 5 , it is likely that pyrophosphate release is coupled to active-site rearrangements that are induced by translocation. Comparison of stage 7 (a pre-translocation state) and stage 5 (equivalent to a post-translocation state) also reveals residues that select and coordinate the incoming NTP. Studying these residues may afford a better understanding of the error rate of influenza virus RNA polymerase $^{13}$ or how mutations in this active site confer resistance to nucleotide analogs, such as favipiravir ${ }^{14}$.

In the three structures discussed above, additional primer-template base pairs form downstream of the initiation site due to extension of the template (Fig. 1, stages 5-7). These extra base pairs are artificial and induce changes that probably do not occur during normal transcription initiation. The complexes are thus in a mixed state. However, the structural rearrangements are nevertheless informative. The most striking example is a remodeling and partial extrusion of the priming loop, which is a structure that reduces the size of the active- site cavity (Fig. 1, stages 1-3) and has a key role in replication initiation ${ }^{15}$. Published experiments with RNA polymerases of influenza virus and other viruses have shown that truncation of the priming loop stimulates elongation ${ }^{15-17}$. The crystals generated by Kouba, Drncova and Cusack ${ }^{5}$ are consistent with those observations and suggest that the priming loop would be displaced to accommodate the natural product-template duplex, which would occupy the same location after one to two rounds of NTP incorporation and translocation.

Addition of ATP and GTP to the mixed post-initiation complex (Fig. 1, stage 7) allows the polymerase to extend the capped primer by five bases, before it pauses when a guanosine in the template enters the +1 site and CTP is required (Fig. 1, stage 8 ). A $3.2 \AA$ cryo-electron microscopy structure of this stalled complex reveals that elongation requires unwinding of the promoter duplex at the top of the polymerase and translocation of the template down the template entry channel ${ }^{5}$. The structure also beautifully shows that the template and growing influenza virus mRNA are separated 9 base pairs downstream of the active site. In particular, PB2 residue Thr207 seems to serve a key role in duplex unwinding, because it stacks onto the last template base of the duplex and prevents formation of a tenth base pair (Fig. 1, stage 8). It is possible that this residue acts like a gate, allowing one template base to pass into the template exit channel before closing again onto the next template base. In addition, it is tempting to speculate that the 9-base duplex confers processivity and limits early dissociation of influenza virus RNA products, which can result in early termination or transcriptional realignment ${ }^{9,18}$.

Elongation also involves conformational changes in the polymerase. Most notable is the complete extrusion of the priming loop, which generates more space in the active-site cavity and ensures that unwound template base(s) can enter the exit channel (Fig. 1 , stage 8 ). In addition, repositioning of 

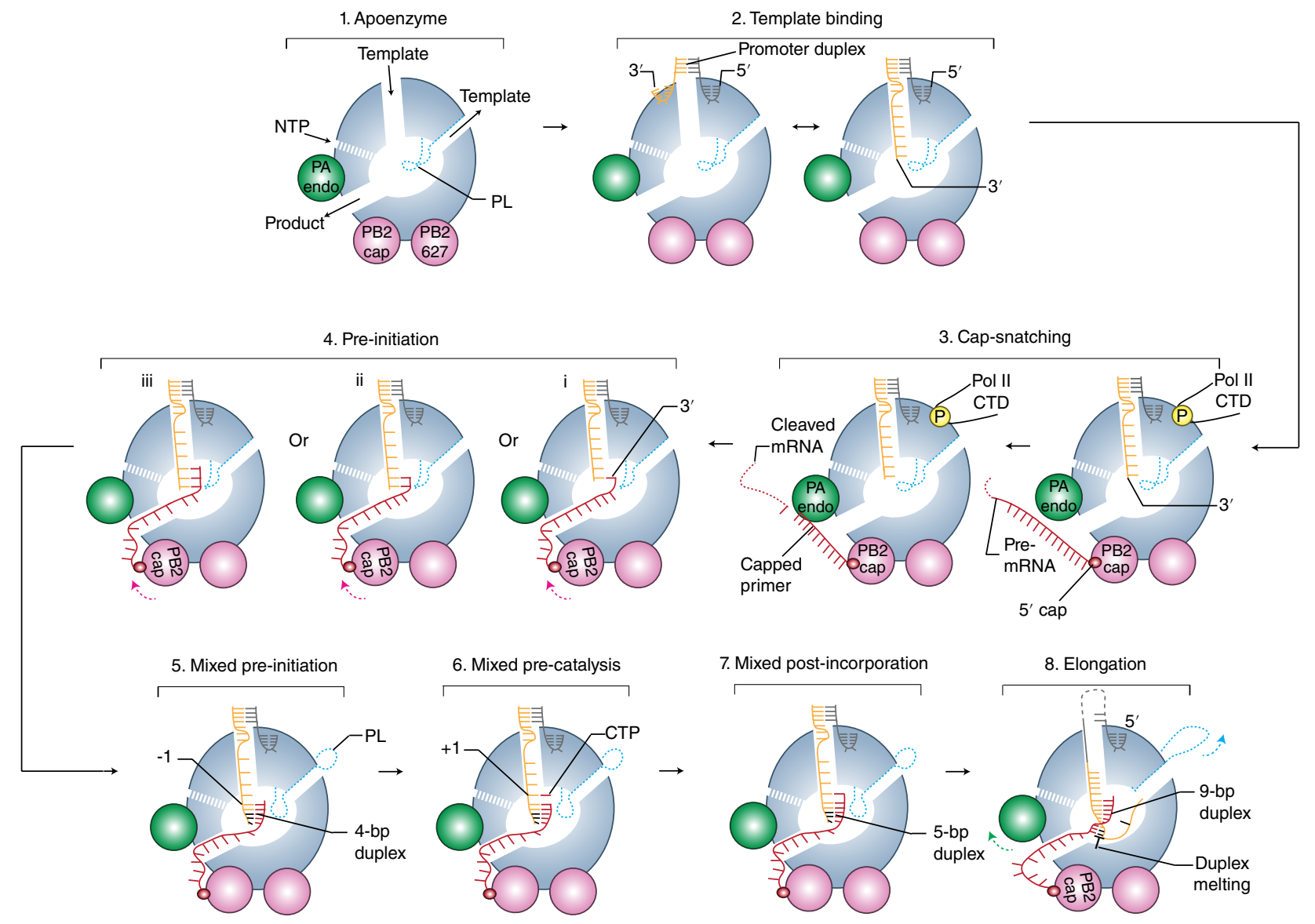

Fig. 1 | Stages of influenza virus transcription initiation. (1) The influenza virus RNA polymerase consists of a central core (blue), the flexible PA endonuclease (PA endo; green), the PB2 cap-binding domain (PB2 cap; pink) and the PB2 627 domain (PB2 627; pink). Channels lead from the solvent to the active site of the polymerase to facilitate the entry or exit of templates, NTPs and products. The priming loop (PL; blue dotted line) occupies part of the active-site cavity. The flexible domains can adopt at least two orientations, but for clarity the apoenzyme is shown in the conformation that is compatible with transcription. (2) The segments that make up the influenza virus genome contain 3' (orange) and 5' (gray) promoter elements that bind to the top of the RNA polymerase. The location of the $3^{\prime}$ terminus is dynamic and varies between binding at the top of the RNA polymerase or threading through the template entry channel. (3) Binding of viral RNA polymerase to Ser5-phosphorylated (P; yellow) C-terminal domain (CTD) residues of initiating eukaryotic polymerase II (Pol II) complexes brings the PB2 cap-binding domain in close proximity to emerging, capped cellular pre-mRNAs (red). The PA endonuclease cleaves these premRNAs 10-14 nucleotides downstream of the cap to generate a capped primer. (4) Reorientation of the PB2 cap-binding domain threads the capped primer into the product exit channel. The capped primer then forms base pairs with the first, second or third base (uridine, cytosine or guanosine, respectively) of the $3^{\prime}$ terminus of the template. (5) In the mixed pre-initiation complex, the extended template (black) forms extra base pairs with a 14-nucleotide-long primer that binds to the second base of the $3^{\prime}$ terminus of the natural template. (6) Addition of CTP (red) to the pre-initiation complex reveals a density in the +1 binding site. (7) The extended template forms 5 base pairs with a 15-nucleotide-long primer. The primer binds to the third base of the $3^{\prime}$ terminus of the natural template. (8) Extension of the capped primer in the presence of only ATP and GTP leads to the formation of a stalled complex, which forms a 9-base pair duplex in the active-site cavity. The duplex is separated at PB2 Thr207 (T). Several rearrangements in the polymerase (dotted arrows) widen the exit channels to allow product and template egress. Further extension will involve translocation of the main part of the viral RNA template (dotted dashed line) and the $5^{\prime}$ promoter.

the thumb subdomain and of PB2 and PA residues helps widen the active-site cavity and polymerase exit channels. These changes all aid a transition to progressive elongation, which will eventually push the template through the exit channel, while the growing mRNA will bulge out of the product exit channel before being released by the PB2 cap-binding domain ${ }^{19}$ and exported to the cytoplasm for translation.

In summary, the work by Kouba,

Drncova and Cusack ${ }^{5}$ constitutes a considerable advance in the understanding of influenza virus transcription. Not only do the new structures and observed conformational changes offer plenty of targets for structure-guided mutagenesis studies and the design of new antivirals, they will probably also aid studies aimed at investigating the dynamics of the polymerase or understanding how aberrant RNA molecules that stimulate the immune response $^{20}$ are produced. Moreover, the methodology used may help further structural studies of influenza virus RNA synthesis.

\section{Aartjan J. W. te Velthuis (D)}

University of Cambridge, Department of Pathology, Addenbrooke's Hospital, Cambridge, UK. e-mail:ajwt6@cam.ac.uk

Published online: 3 June 2019

https://doi.org/10.1038/s41594-019-0243-9

\section{References}

1. Krug, R. M., Morgan, M. A. \& Shatkin, A. J. J. Virol. 20, 45-53 (1976).

2. Etkind, P. R. \& Krug, R. M. Virology 62, 38-45 (1974).

3. Decroly, E. \& Canard, B. Curr. Opin. Virol. 24, 87-96 (2017). 
4. Plotch, S. J., Bouloy, M., Ulmanen, I. \& Krug, R. M. Cell 23, 847-858 (1981).

5. Kouba, T., Drncova, P. \& Cusack, S. Nat. Struct. Mol. Biol. https:// doi.org/10.1038/s41594-019-0232-z (2019).

6. Lukarska, M. et al. Nature 541, 117-121 (2017).

7. Serna Martin, I. et al. Mol. Cell 70, 1101-1110.e4 (2018).

8. Reich, S. et al. Nature 516, 361-366 (2014).

9. Koppstein, D., Ashour, J. \& Bartel, D. P. Nucleic Acids Res. 43, 5052-5064 (2015).

10. Reich, S., Guilligay, D. \& Cusack, S. Nucleic Acids Res. 45 3353-3368 (2017).

11. Robb, N. C. et al. Nucleic Acids Res. 44, 10304-10315 (2016).
12. Robb, N., te Velthuis, A.J.W., Fodor, E. \& Kapanidis, A.N. Nucleic Acids Res. https://doi.org/10.1093/nar/gkz313 (2019).

13. Pauly, M. D., Procario, M. C. \& Lauring, A. S. eLife 6, e26437 (2017).

14. Goldhill, D. H. et al. Proc. Natl Acad. Sci. USA 115, 11613-11618 (2018).

15. te Velthuis, A. J. W., Robb, N. C., Kapanidis, A. N. \& Fodor, E. Nat. Microbiol. 1, 16029 (2016).

16. Appleby, T. C. et al. Science 347, 771-775 (2015).

17. Laurila, M. R. L., Makeyev, E. V. \& Bamford, D. H. J. Biol. Chem. 277, 17117-17124 (2002).

18. Klumpp, K., Ford, M. J. \& Ruigrok, R. W. J. Gen. Virol. 79 , 1033-1045 (1998).
19. Braam, J., Ulmanen, I. \& Krug, R. M. Cell 34, 609-618 (1983). 20. Te Velthuis, A. J. W. et al. Nat. Microbiol. 3, 1234-1242 (2018).

Acknowledgements

A.J.W.t.V. is supported by joint Wellcome Trust and Royal Society grant 206579/Z/17/Z and Isaac Newton Trust grant 17.37(r).

Competing interests

The author declares no competing interests.

\title{
Insights into L-type heteromeric amino acid transporters
}

\author{
Two recent cryo-EM structures of the human L-type heteromeric amino acid transporter LAT1-CD98hc reveal \\ surprising new insights into both amino acid transport in the human body and the roles of CD98hc as a \\ cell-surface antigen and trafficking chaperone.
}

\section{Simon Newstead}

W hile amino acids are familiar to many as the building blocks of proteins, they also serve essential roles in regulating cellular metabolism (Fig. 1). Proliferating cells need additional amino acids as precursors in metabolic pathways and to stimulate the metabolic checkpoint kinase complex mTORC1 (mechanistic target of rapamycin complex 1), which functions to increase anabolic processes ${ }^{1}$. Cytoplasmic amino acid pools are controlled by several families of plasmamembrane amino acid transporters, which makes these proteins important regulators of cell growth that are upregulated in rapidly proliferating cancer cells and, hence, they are important targets in ongoing anti-cancer drug-discovery programs ${ }^{2}$.

Mammalian amino acid transporters are categorized into several transport systems on the basis of their substrate selectivity and mechanism. The uptake and distribution of dietary amino acids is carried out predominantly by so-called 'system- $\mathrm{L}$ transporters', which mediate $\mathrm{Na}^{+}$-independent transport of large amino acids across the plasma membrane ${ }^{3}$. Among these, LAT1 (L-type amino acid transporter 1; also known as SLC7A5) has attracted the most interest due to its prominent role in stimulating cancer-cell growth through the uptake of L-leucine ${ }^{4}$, supplying thyroid hormones to developing fetuses and transporting L-DOPA (L-3,4-dihydroxyphenylalanine) and other neurological drugs across the blood-brain barrier ${ }^{5}$. As a subunit of a heteromeric amino acid transporter, LAT1, referred to as the 'light' subunit, associates with a second protein, CD98hc (also known as $4 \mathrm{~F} 2 \mathrm{hc}$ or SLC3A2), referred to as the 'heavy' subunit. CD98hc, with molecular weight of $85 \mathrm{kDa}$ in its mature form, comprises a single transmembrane domain and a large extracellular domain that is heavily glycosylated ${ }^{6}$.

For several years, understanding of the structure of the LAT1-CD98hc heteromeric complex was gleaned from the crystal structures of distantly related bacterial homologs of the transport domain ${ }^{7-11}$ and an isolated crystal structure of the glycoprotein minus the transmembrane segment ${ }^{6}$. Several key questions about the function of mammalian LAT1 have therefore remained unanswered, such as how the light and heavy subunits interact in the membrane, the structural basis of amino acid specificity and an accurate structural model of the binding site to advance structure- and computationbased design of inhibitors and/or drugs.

Now, using single-particle cryo-EM, two groups have independently reported structures of the human LAT1-CD98hc complex $^{12,13}$. LAT1 is captured in an inward open conformation, with the binding site accessible to the inside of the cell (Fig. 2a). Nureki and colleagues report a structure in complex with two antibodies to CD98hc that are currently being evaluated as anti-cancer agents $^{12}$. Zhou and colleagues report a complex with the competitive inhibitor $\mathrm{BCH}$ ((1S,2S,4R)-2-aminobicyclo[2.2.1] heptane-

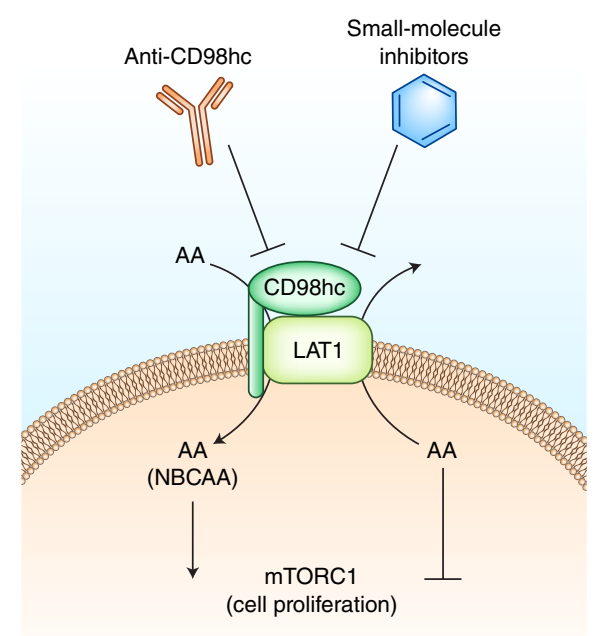

Fig. 1 | Physiological role of the LAT1-CD98hc heteromeric amino acid transporter. LAT1 regulates $\mathrm{mTORC} 1$ activity by controlling the cytoplasmic concentrations of neutral branchedchain amino acids (NBCAA) at the plasma membrane of eukaryotic cells. Several strategies are being developed to inhibit LAT1 in an effort to develop new anti-cancer therapies. These involve either antibodies targeted to CD98hc

(Anti-CD98hc) or small-molecule inhibitors of LAT1 transport.

2-(arboxylic acid) $)^{13}$, which functions as an anti-cancer molecule in tissue culture ${ }^{14}$. Both studies provide valuable insights into ligand 